\title{
Les vaccins HPV provoquent-ils des maladies neurologiques?
}

\author{
Heinrich P. Mattlea, Valeriu Toma ${ }^{b}$, Rudolf Stoller \\ ${ }^{\text {a }}$ Clinique universitaire de neurologie, Inselspital, Berne; b Swissmedic, Institut suisse des produits thérapeutiques, Berne
}

\section{Synthèse}

Les papillomavirus humains (HPV) provoquent des cancers du col de l'utérus, des verrues génitales ainsi que plus rarement des carcinomes au niveau de la vulve, de l'anus, du pénis, de la base de la langue et de la gorge. Or la vaccination contre ces virus, qui sont transmis lors des rapports sexuels, peut prévenir efficacement le cancer du col de l'utérus. Les études épidémiologiques réalisées n'ont pas confirmé les craintes $\mathrm{au}$ sujet d'effets secondaires neurologiques graves (sclérose en plaques, syndrome douloureux régional complexe ou syndrome de tachycardie orthostatique posturale). Le seul effet secondaire de ce type qui a été observé plus fréquemment chez les personnes qui avaient été vaccinées contre les HPV (1,4 cas pour 100000 patients par an) que chez celles qui n'avaient pas été vaccinées $(0,4$ cas pour 100000 patients par an), selon une nouvelle étude française, concerne les polyradiculonévrites, mais il s'agit là d'un premier résultat qui doit être confirmé par des analyses supplémentaires. Cette augmentation potentielle du risque est par ailleurs à mettre en balance avec le bénéfice du vaccin HPV, à savoir la prévention de maladies et de décès dus aux papillomavirus humains.

\section{Pertinence de la vaccination contre les HPV}

En 2008, Harald zur Hausen s'est vu décerner le Prix Nobel de médecine. En 1976, il avait avancé l'hypothèse selon laquelle des papillomavirus jouaient un rôle pathogénique dans l'apparition du cancer du col de l'utérus, hypothèse qu'il a confirmée en 1983-84 en démontrant la présence d'ADN du HPV 16 et du HPV 18 dans ces tumeurs [1]. Telle fut la pierre angulaire sur laquelle s'appuieront le développement d'un premier vaccin et l'introduction de ce dernier en 2006. Les filles et les jeunes femmes constituent le principal groupe cible du vaccin HPV, qui doit être administré idéalement avant les premières relations sexuelles. Selon les données de l'Agence européenne du médicament (EMA), plus de 80 millions de personnes ont été vaccinées à ce jour, et dans les pays où le taux de vaccination est élevé (Australie, Danemark, Suède et Etats-Unis), les maladies in- duites par les HPV, dont les verrues génitales et les anomalies du col de l'utérus, ont diminué sensiblement chez les jeunes. Or à l'heure actuelle, 20000 femmes décèdent chaque année en Europe des suites d'un cancer du col de l'utérus. En Suisse, l'Institut National pour l'Epidémiologie et l'Enregistrement du Cancer a recensé 90 décès suite à des carcinomes du col de l'utérus ainsi que 231 nouvaux cas de ce cancer en 2012 [2]. On peut espérer que la vaccination contre les HPV permettra de faire diminuer ces chiffres, mais il faudra encore patienter un certain temps avant de pouvoir constater un effet visible en ce sens, car en règle générale, il s'écoule plus d'une vingtaine d'années entre l'infection par le HPV et le développement de la tumeur maligne [3]. La majorité des personnes ont été infectées lorsqu'elles étaient jeunes, et sont confrontées à des conséquences majeures.

\section{Préparations autorisées en Suisse}

C'est fin 2006 que le premier vaccin contre les HPV a été autorisé simultanément en Suisse et dans d'autres pays à travers le monde. Il s'agissait du vaccin quadrivalent Gardasil $^{\circledR}$, qui est actif contre les types 16 et 18 des HPV, tumorigènes et responsables, selon les données actuelles, d'environ $70 \%$ des cas de carcinomes du col utérin, ainsi que contre les types 6 et 11, qui occasionnent des verrues génitales. Le vaccin Cervarix ${ }^{\circledR}$, qui a été autorisé au printemps 2010, agit uniquement contre les types 16 et 18 des HPV, et ne prévient donc que les tumeurs. L'Office fédéral de la santé publique recommande d'effectuer la vaccination à un âge précoce, soit entre 11 et 14 ans. Le vaccin n'a aucun effet sur les infections par les HPV acquises avant l'injection, mais il protège face aux autres types de HPV qui sont couverts par le vaccin.

\section{Effets indésirables liés aux vaccins HPV}

L'augmentation du nombre de vaccins HPV administrés a également fait croître le nombre d'annonces individuelles et de publications au sujet de séries de cas qui font état de maladies et d'effets secondaires potentiels en lien temporel avec la vaccination. Ces publications 
font naître des craintes au sujet d'effets néfastes de ces vaccins, et en particulier de maladies du système nerveux central et périphérique (sclérose en plaques ou polyradiculonévrite de Guillain-Barré). D’autres complexes de symptômes tels que le syndrome douloureux régional complexe (maladie de Sudeck, algodystrophie, SDRC) ou celui de la tachycardie orthostatique posturale (STOP) ont été reliés avec la vaccination contre les HPV sur le plan chronologique. Cependant, ces rapports et séries de cas signalés par des centres spécialisés ne permettent pas d'établir un lien de causalité entre ces maladies et syndromes d'une part et la vaccination d'autre part; et en réalité, au niveau individuel, le rôle causal du vaccin ne peut être ni confirmé ni exclu. Par ailleurs, en ce qui concerne les séries de cas déclarés par des centres spécialisés (relatifs au STOP par exemple) surtout, l'on observe un biais de sélection au niveau des groupes de patients présentant les symptômes en question. Or un lien de causalité ne peut être démontré que lorsque des études épidémiologiques menées à grande échelle mettent en évidence un risque relatif significativement plus élevé chez les personnes qui ont été vaccinées par rapport à celles qui ne l'ont pas été. Par conséquent, le fait que des études dont la puissance statistique («statistical power») est suffisante ne révèlent pas de différences significatives au niveau de l'incidence d'une maladie chez les personnes qui ont été vaccinées et chez celles qui ne l'ont pas été réfute l'existence d'un lien de causalité éventuellement présumé.

\section{Profil de sécurité des vaccins HPV}

Depuis la mise sur le marché de ces vaccins, leurs risques font l'objet d'une surveillance et d'un suivi étroits en Suisse et à travers le monde, ainsi qu'au niveau de l'Organisation mondiale de la santé (OMS). Depuis lors, l'expérience accumulée avec les deux préparations est considérable. En Suisse, c'est surtout la préparation Gardasil ${ }^{\circledR}$ qui a été suivie de près, puisque fin 2015, soit en neuf ans, environ 900000 doses de ce vaccin avaient été distribuées dans notre pays. A travers le monde, ce sont plus de 200 millions de doses qui avaient déjà été administrées à cette date [4].

Les douleurs ou les signes d'inflammation au niveau du site d'injection, les céphalées, la fièvre et les symptômes grippaux (douleurs au niveau des membres notamment) font partie des effets indésirables (EI) assez fréquents et bien connus pour ces préparations. Ces symptômes, qui apparaissent peu de temps après l'injection pour disparaître en général spontanément, sont des effets indésirables classiques des vaccins. Compte tenu de données internationales recueillies dans le cadre de la surveillance après la mise sur le marché, l'information professionnelle de Gardasil ${ }^{\circledR}$ mentionne divers EI (encéphalomyélite aiguë disséminée [ADEM], polyradiculonévrite [syndrome de Guillain-Barré, SGB], névrite optique, syncopes, vertiges, malaise général et fatigue). Dans la mesure où ces EI proviennent d'annonces spontanées, il n'a pas été possible d'évaluer de manière fiable leur fréquence exacte ou leur lien de causalité avec le vaccin lors de leur inclusion dans l'information professionnelle [5].

\section{Annonces d'EI survenus en Suisse}

De 2007 à ce jour (état de la base de données en décembre 2015), Swissmedic a reçu au total 184 déclarations suisses d'EI présumés impliquant des vaccins HPV, dont 177 au sujet de Gardasil ${ }^{\circledR}$, ce qui pourrait être imputé aux parts de marché plus importantes de cette préparation. Depuis 2010, l'institut reçoit entre 10 et 30 déclarations par an. Un maximum a été atteint en 2009 (95 déclarations), car des événements mineurs qui n'étaient pas soumis à l'obligation de déclarer ont aussi été saisis systématiquement au niveau régional et transmis à Swissmedic.

Sur les 184 annonces, 61\% furent considérées comme faisant état d'EI «non graves», tandis que $27 \%$ décrivaient des EI «médicalement importants». Dans 12\% des cas, les conséquences furent graves, avec des hospitalisations notamment, mais aucun décès n’a été déclaré.

Ces déclarations laissent donc penser que le profil de sécurité de ces vaccins est favorable et correspond aux risques qui sont mentionnés de manière détaillée dans l'information sur le médicament [5]. Suite à des déclarations de syncopes vasovagales survenues immédiatement après l'administration du vaccin, Swissmedic et l'OFSP ont ordonné sur le champ de nouvelles mesures de précaution [6], selon lesquelles les patientes doivent être assises ou couchées pendant l'administration du vaccin, puis être surveillées pendant une quinzaine de minutes. Cela étant, les syncopes, suivies parfois de chutes, peuvent survenir après ou même avant n'importe quelle injection, surtout chez les jeunes, et peuvent s'accompagner d'autres symptômes neurologiques (troubles passagers de la vision, paresthésies, et même mouvements tonico-cloniques des membres). Les syncopes vasovagales restent sans danger pour autant qu'on évite les chutes et les blessures qui pourraient en résulter.

Annonces de démyélinisations: A ce jour, Swissmedic n'a reçu qu'une seule déclaration provenant de Suisse au sujet d'une sclérose en plaques apparue après une injection de Gardasil ${ }^{\circledR}$. Et une autre patiente, qui avait déjà eu une névrite optique avant la première injection de Cervarix ${ }^{\circledR}$, a contracté une myélite après cette der- 
nière. D'après l'analyse neurologique, cette myélite était donc une deuxième manifestation clinique d'une maladie démyélinisante (sclérose en plaques par exemple), et l'apparition de cette maladie datait d'avant l’administration de Cervarix ${ }^{\circledR}$.

En Suisse, on dénombre quatre à six nouveaux cas de sclérose en plaques par 100000 habitants chaque année, étant entendu que les jeunes femmes, qui constituent le groupe cible des vaccins contre les HPV, sont deux fois plus touchées que les hommes. Or s'il existait un lien de causalité entre les vaccins HPV et la sclérose en plaques, il faudrait s'attendre, compte tenu des 900000 et quelques injections effectuées en Suisse à ce jour, à un nombre de cas bien plus élevé que ceux évoqués dans les deux déclarations dont il est question ci-dessus.

Certaines annonces suisses faisaient état de symptômes neuropsychiatriques divers: asthénie, épuisement, fatigue ou malaise général. Toutefois, pour une écrasante majorité des personnes concernées, l'annonce de l'EI documentait déjà le rétablissement total du patient au moment de l'envoi de la déclaration.

Aucun cas d'ADEM ou de SGB en rapport avec des vaccins HPV n’a été déclaré à ce jour en Suisse.

\section{Etudes sur la sécurité des préparations après octroi des autorisations de mise sur le marché}

Sclérose en plaques et autres maladies démyélinisantes du système nerveux central

$\mathrm{Au}$ Danemark et en Suède, il est possible d'établir un lien entre le statut vaccinal d'une personne et l'apparition d'une maladie démyélinisante au niveau individuel en s'appuyant sur un numéro d'identification personnel et sur divers registres. Il est ainsi possible de déterminer si une personne a présenté des symptômes d'une maladie démyélinisante après l'administration du vaccin et, le cas échéant, quand. Dans une étude de cohorte réalisée entre 2006 et 2013, Scheller et ses collaborateurs ont analysé l'apparition d'une maladie démyélinisante ainsi que le statut vaccinal et la date de vaccination de 3983824 jeunes filles et femmes, âgées de 10 à 44 ans, et ont donc ainsi passé en revue dans leur étude 21332622 années-patients [7]. Les maladies démyélinisantes comprenaient la sclérose en plaques et d'«autres affections démyélinisantes»: névrites optiques, myélites transverses, syndromes cliniques isolés, encéphalomyélites aiguës disséminées et neuromyélites optiques. Dans plus de 99\% des cas, le produit utilisé était le vaccin quadrivalent Gardasil ${ }^{\circledR}$, et la période à risque sélectionnée correspondait aux deux ans qui suivaient la dernière injection. Différentes comparaisons statistiques entre la cohorte vaccinée et celle qui ne l'était pas n'ont révélé aucune différence significative au niveau de l'incidence de la sclérose en plaques ou d'autres maladies démyélinisantes. Dans une autre analyse, d'une "self-controlled case series", des jeunes filles et des femmes vaccinées ont été utilisées comme leur propre contrôle, dans la mesure où l'on a comparé l'incidence de ces maladies pendant la période qui avait précédé et celle qui avait suivi la vaccination. Ici encore, aucune différence n'a été observée. Pour la sclérose en plaques et d'autres maladies démyélinisantes, les risques relatifs étaient de 1,05 (0,79-1,38 avec un intervalle de confiance de 95\%) et de 1,14 (0,88-1,47 avec un intervalle de confiance de 95\%). Cette étude à grande échelle et de très bonne qualité sur le plan scientifique démontre qu'il n'existe aucun lien de causalité entre les vaccins HPV quadrivalents et la sclérose en plaques ou d'autres maladies démyélinisantes. Les travaux réalisés en France dont il est question ci-dessous ainsi qu'une étude californienne ont d'ailleurs abouti à la même conclusion [8, 9].

\section{Polyradiculonévrite de Guillain-Barré}

Des auteurs français ont mené une étude de cohorte en suivant une méthode similaire à celle de l'étude suédo-danoise [7, 8]. La question était de savoir si des maladies auto-immunes de manière générale et en particulier de telles maladies apparaissaient plus fréquemment après administration du vaccin HPV. Les auteurs ont inclus 2252716 jeunes filles âgées de 13 à 16 ans entre début 2008 et fin 2012, et ont ainsi passé en revue 6139497 années-patients. Au total, 93\% des injections avaient été réalisées avec le vaccin quadrivalent Gardasi ${ }^{\circledR}$, et $7 \%$ avec le vaccin bivalent Cervarix ${ }^{\circledR}$. Sur les 29 maladies inflammatoires examinées, un seul résultat clairement significatif est apparu, par rapport à la polyradiculonévrite de Guillain-Barré (hazard ratio de 4,0; 1,84-8,69 avec un intervalle de confiance de $95 \%$; $p<0,001)$, ce risque étant particulièrement accru dans les trois mois qui suivaient la vaccination. Sur 100000 patients, le taux de polyradiculonévrites était d'un cas de plus par an après la vaccination qu'avant ou sans cette dernière. Il s'agit en l'occurrence de la première analyse épidémiologique suggérant un lien entre les vaccins HPV et les polyradiculonévrites. Ces résultats doivent toutefois être confirmés par d'autres analyses, d'autant que des études effectuées dans le passé, qui portaient sur les vaccins HPV ou autres, n'ont pas mis en exergue de risque accru en ce qui concerne le SGB [10,11]. Et en Californie, aucun cas nouveau de polyradiculonévrite n'a été identifié pour près de 190000 femmes vaccinées avec Gardasil ${ }^{\circledR}$ [12].

\section{Syndrome douloureux régional complexe - SDRC (complex regional pain syndrome, CRPS)} Le SDRC est un syndrome douloureux qui évolue lente- 
ment et qui apparaît typiquement après un traumatisme ou des interventions chirurgicales. Des déclarations de SDRC après administration d'un vaccin HPV au Japon ont conduit à la suspension temporaire de la recommandation vaccinale. Mais une étude réalisée par la suite avec des données japonaises et anglaises n'a pas confirmé l'existence d'un lien entre le SDRC et le vaccin Cervarix ${ }^{\circledR}$ [13]. Eu égard aux 17 cas avérés de SDRC après administration d'un vaccin HPV au Japon et en Angleterre, un groupe d'experts a calculé que l'incidence de ce syndrome par dose de vaccin distribuée était inférieure à l'incidence de fond à laquelle il fallait s'attendre. L'étude susmentionnée n'apporte donc aucune preuve que les vaccins HPV provoquent un SDRC. Le Comité pour l'évaluation des risques en matière de pharmacovigilance (PRAC) de l'Agence européenne du médicament a d'ailleurs abouti à la même conclusion [14].

\section{Syndrome de tachycardie orthostatique postu- rale-STOP (postural orthostatic tachycardia syndrome, POTS)}

Suite à quelques rapports et séries limitées de cas d'hypotension orthostatique après vaccination contre les HPV, d'aucuns se sont interrogés quant à l'existence éventuelle d'un lien de causalité entre ces éléments. Des tests d'inclinaison réalisés auprès d'une série de 35 patients souffrant d'intolérance orthostatique après vaccination avec Gardasil ${ }^{\circledR}$ ont mis en lumière un syndrome STOP chez 60\% d'entre eux [15]. Mais comme pour le SDRC, le rapport d'expertise de l'EMA n'a identifié aucun lien [14]. Chaque année, au moins 150 cas de syndrome STOP pour un million de personnes sont observés chez des jeunes filles. Et ce chiffre n'est pas plus élevé chez celles qui ont été vaccinées contre le HPV, ce qui affaiblit la présomption d'un lien entre la vaccination contre les HPV et le syndrome STOP.

\section{Syndrome de fatigue chronique - SFC (chronic fatigue syndrome, CFS)}

Dans le cadre du programme vaccinal anglais, une série d'effets secondaires qui avaient un lien chronologique avec les vaccinations, dont le SFC, ont été saisis systématiquement. Mais en l'occurrence, le nombre de cas observés après l'injection de Cervarix ${ }^{\circledR}$ n'était pas plus élevé que le taux attendu au niveau de la population non vaccinée (incident rate ratio: 0,94; avec un intervalle de confiance de 95\% entre 0,78-1,14) [16]. De plus, il n'y avait aucune différence au niveau de la fréquence des SFC avant et après la vaccination (incident rate ratio: 1,07; avec un intervalle de confiance de $95 \%$ entre 0,57-2,00). Selon cette étude, il n'existe aucun lien de cause à effet entre les vaccins HPV et le SFC.

\section{Conclusion sur les données disponibles actuellement}

Les annonces individuelles et les séries de cas qui font état d'effets secondaires ou de maladies apparues juste après l'administration d'un vaccin ou d'un médicament peuvent fournir des informations importantes au sujet de la sécurité d'emploi des produits en question. Elles servent à saisir des signaux de sécurité, à savoir de nouveaux risques présumés ou de nouveaux aspects de risques connus. Toutefois, la plupart du temps, ces données ne permettent ni de confirmer ni d'infirmer un éventuel lien de cause à effet. Pour ce faire, des études épidémiologiques à plus grande échelle doivent être réalisées afin de comparer des cohortes de population. De grandes séries de cas avec contrôle interne (selfcontrolled case series) peuvent également fournir des informations fiables. Depuis la mise sur le marché du premier vaccin HPV dans le monde en 2006, de vastes quantités de données ont été recueillies et analysées, et ces données montrent qu'à ce jour, il n'y a aucune raison de penser que ces vaccins pourraient provoquer des maladies du système nerveux central. Elles ne montrent pas la présence d'une causalité entre la vaccination contre les HPV et les scléroses en plaques ou d'autres maladies cérébrales démyélinisantes. Au niveau du système nerveux périphérique en revanche, une nouvelle étude a mis en évidence pour la première fois une augmentation du nombre de cas de polyradiculonévrites de Guillain-Barré après vaccination contre les HPV, mais ce résultat, qu'il y a lieu d'interpréter prudemment, selon les auteurs de l'étude, doit être confirmé par d'autres analyses. De plus, il faut savoir que grâce aux possibilités thérapeutiques actuelles, la plupart des personnes atteintes de polyradiculonévrite guérissent sans séquelle. En chiffres absolus, le risque correspond environ, pour 100000 années-patients, à un cas de plus que chez les personnes non vaccinées. Cette augmentation potentielle minime du risque doit donc être mise en balance avec le bénéfice des vaccins contre les HPV, qui permettent d'éviter un grand nombre de maladies et de décès dus aux papillomavirus humains. Faire vacciner les adolescents contre les HPV permettrait d'ailleurs probablement d'éviter la plupart des affections dues au HPV à l'avenir, et les données actuelles sur les EI étayent plus que jamais la sécurité de ce vaccin.

Tant en Suisse qu'à l'étranger, ces derniers continuent de faire l'objet d'une surveillance étroite. Les professionnels de santé sont donc invités à transmettre leurs annonces d'effets indésirables suspectés à Swissmedic en utilisant le portail électronique ElVis (www.swiss medic.ch $\rightarrow$ Surveillance du marché $\rightarrow$ Pharmacovigilance $\rightarrow$ ElViS). 


\section{Références}

1 www.nobelprize.org/nobel_prizes/medicine/laureates/2008/ hausen-facts.html (page consultée pour la dernière fois le 5 février 2016)

2 www.nicer.org (page consultée pour la dernière fois le 5 février 2016).

3 Castle PE, Maza M. Prophylactic HPV vaccination: past, present, and future. Epidemiol Infect. 2016;144:449-68.

4 Comité consultatif mondial sur la sécurité des vaccins: Déclaration du GACVS sur l'innocuité des vaccins contre le papillomavirus humain (PVH) - 17 décembre 2015.

5 www.swissmedicinfo.ch (page consultée pour la dernière fois le 5 février 2016).

6 Slade BA, Leidel L, Vellozzi C, Woo EJ, Hua W, Sutherland A, et al. Postlicensure safety surveillance for quadrivalent human papillomavirus recombinant vaccine. JAMA 2009;302:750-7.

7 Scheller NM, Svanström H, Pasternak B, Arnheim-Dahlström L, Sundström K, Fink K, Hviid A. Quadrivalent HPV vaccination and risk of multiple sclerosis and other demyelinating diseases of the central nervous system. JAMA. 2015;313:54-61.

8 Alpérovitch A, Elbaz A, Faye A, Fournier A, Galanaud P, Heard I, Bader-Meunier B, Mrejen S. ANSM/CNAMTS -Vaccin anti-HPV et risque de maladies auto-immunes: étude pharmacoépidémiologique. Rapport final, septembre 2015. http://ansm.sante.fr/ content/download/80841/1023043/version/1/file/Ansm GardasilHpv2_Rapport_Septembre-2015.pdf

9 Langer-Gould A, Qian L, Tartof SY, Brara SM, Jacobsen SJ, Beaber BE, Sy LS, Chao C, Hechter R, Tseng HF. Vaccines and the risk of multiple sclerosis and other central nervous system demyelinating diseases. JAMA Neurol. 2014;71:1506-13.
10 Ojha RP, Jackson BE, Tota JE, Offutt-Powell TN, Singh KP, Bae S. Guillain-Barré syndrome following quadrivalent human papillomavirus vaccination among vaccine-eligible individuals in the United States. Hum Vaccin Immunother. 2014;10:232-7.

11 Baxter R, Bakshi N, Fireman B, Lewis E, Ray P, Vellozzi C, Klein NP. Lack of association of Guillain-Barré syndrome with vaccinations. Clin Infect Dis. 2013;57:197-204

12 Chao C, Klein NP, Velicer CM, Sy LS, Slezak JM, Takhar H, Ackerson B, Cheetham TC, Hansen J, Deosaransingh K, Emery M, Liaw KL, Jacobsen SJ. Surveillance of autoimmune conditions following routine use of quadrivalent human papillomavirus vaccine. J Intern Med. 2012;271:193-203.

13 Huygen F, Verschueren K, McCabe C, Stegmann JU, Zima J, Mahaux O, Van Holle L, Angelo MG. Investigating Reports of Complex Regional Pain Syndrome: An Analysis of HPV-16/18-Adjuvanted Vaccine Post-Licensure Data. EBioMedicine. 2015 Jul 6;2(9):1114-21.

14 European Medicines Agency. HPV vaccines: EMA confirms evidence does not support that they cause CRPS or POTS. http:// www.ema.europa.eu/docs/en GB/document library/Referrals document/HPV_vaccines_20/Opinion_provided_by_Committee for_Medicinal Products for Human Use/WC500196773.pdf (page consultée pour la dernière fois le 5 février 2016).

15 Brinth LS, Pors K, Theibel AC, Mehlsen J. Orthostatic intolerance and postural tachycardia syndrome as suspected adverse effects of vaccination against human papilloma virus. Vaccine. 2015;33:2602-5.

16 Donegan K, Beau-Lejdstrom R, King B, Seabroke S, Thomson A, Bryan P. Bivalent human papillomavirus vaccine and the risk of fatigue syndromes in girls in the UK. Vaccine. 2013;31:4961-7. 